\title{
Interactive comment on "Correlation between subsurface salinity anomalies in the Bay of Bengal and the Indian Ocean Dipole and governing mechanisms" by Zheen Zhang et al.
}

Zheen Zhang et al.

zheen.zhang@uni-hamburg.de

Received and published: 26 October 2020

We sincerely thank you for your review. Our response is attached as a PDF file.

Please also note the supplement to this comment:

https://os.copernicus.org/preprints/os-2020-75/os-2020-75-AC2-supplement.pdf 